\title{
Access of Family Planning Services among Urban Poor Women in the City of Yogyakarta
}

\author{
Ratu Matahari", Fitriana Putri Utami, Sri Sugiharti and Sari Kistiana \\ Faculty of Public Health, Universitas Ahmad Dahlan, Warungboto, Kota Yogyakarta 55164, Indonesia
}

('Corresponding author's e-mail: ratu.matahari@ikm.uad.ac.id)

Received: 31 May 2020, Revised: 24 April 2021, Accepted: 30 April 2021

\begin{abstract}
Aim: The barrier factors of family planning programs are divided into individual, household and community level factors. Urbanization and poverty are also conditions related to lack of access to family planning programs. According to the explanation of Green's theory, the behavior of individuals or groups in accessing health services is determined by 3 factors: Predisposing, enabling and reinforcing factors. This study aimed to explain the factors related to family planning programs among poor couples in the city of Yogyakarta. Subject and method: This survey used stratification and multistage random sampling to select the required sample. The units of analysis in this study were women of childbearing age $(15-49$ years), residing in the districts of Tegalrejo, Mergangsan and Umbulharjo, which are poor areas in the city of Yogyakarta. The sample size was 247 women. Results: The factor related to the selection of places to gain access to family planning services for the urban poor in Yogyakarta City is peer support, with a $p$ value $=0.028$. Conclusions: the peer support factor is a factor that is significantly related to the selection of access to family planning services for the urban poor in the city of Yogyakarta. It is recommended to form a community group or local institution that focuses on contraception, so that it can become a form of education and a support system.
\end{abstract}

Keywords: Access, Family planning, Urban poor, Women, Yogyakarta

\section{Introduction}

Indonesia is the $4^{\text {th }}$ highest ranked country in terms of population, with more than 261 million inhabitants in 2016. The rate of population growth has increased sharply in urban areas, with an increase of $11 \%$ in 10 years (as much as $31 \%$ in 1990, $42 \%$ in 2000, and $50 \%$ in 2010, and which is expected to increase by $57 \%$ in 2020). Data from the National Development Planning Agency (Bappenas) explains that urban living in Indonesia has increased, with an indicator of ownership of basic sanitation facilities at $56 \%$ in 2011, even though these conditions have not reached the 2015 target of $69 \%$. The number of poor people has also declined from $16 \%$ in 2007 to $12 \%$ in 2011 [1]. Yogyakarta City, as a research location, is an area with a population of around 2.5 million, with $16 \%$ of the total population below the poverty line $[1,2]$.

Poverty (economic problems) is one of the factors that affect the rate of population growth (fertility), along with education, religiosity, use of contraceptive methods and family planning programs [3]. Poverty and population correlate with family size. A study in Uganda explained that reducing the number of family members can increase family income and improve the quality of children. Another study also explained that the total fertility rate (TFR) is more strongly related to education than economic situation, but that family economy is related to the quality of meeting the reproductive needs of a family [4]. Economy is related to the ability of individuals to access family planning services, so that it has an impact on reducing maternal and child mortality rates [5]. A strategy to improve the quality of life for married couples or women of childbearing age is a family planning program. Family planning programs are very effective in reducing the number of maternal and child deaths; however, poverty is an inhibiting factor in family planning programs, meaning that the poor are often an unmet need group [6].

A study in Uganda explained that poverty makes the poor vulnerable to unmet need [7]. This is supported by the results of a study in Malawi, which explained that groups with high wealth status use modern contraceptives more than poor groups do [7]. The method of contraception is a way to limit the number of children or provide birth spacing. A study explained that increasing the number of family planning acceptors in several developing countries has implications for decreasing maternal mortality cases [8]. The use of contraception is an indicator of global family planning program achievements. The number of family planning users throughout the world increased sharply from $35 \%$ in 1970 to $63 \%$ in 
2017 [9]. This is supported by data from the 2017 Indonesian Health Democratic Survey (IDHS), which explained that as many as $64 \%$ of women of childbearing age aged 15 - 49 use contraception; $57 \%$ use modern contraception, and $6 \%$ use non-modern contraception. A total of $13 \%$ women of childbearing age in Indonesia use long-term contraceptive methods, namely IUDs, implants, and tubectomy (MoW). $29 \%$ of women in childbearing age in Indonesia discontinued use of contraception on the grounds of side effects. The number of unmet needs was explained by $11 \%$ in 2017 [10]. This fact explains that the family planning program is still a challenge in developing countries, including in Indonesia [11].

Indonesia is a country with strong religious and cultural values, so the use of contraception is interpreted as a form of opposition to these values. The values are so strongly related to the concept that a child is a fortune from God that having many children is perceived as the amount of sustenance a family will receive, and the belief that a child is an opening door for sustenance for a family makes a barrier to contraceptive use [12,13]. A study in Pakistan also explained that economic levels and strong religious and cultural norms were also barriers to the use of contraceptive methods [14]. In addition, several studies explained the barrier factors of family planning programs, including individual, household, and community level factors. Urbanization and poverty are also conditions related to lack of access to family planning programs $[5,14,15]$.

Based on the Indonesian Health Demographic Survey (IDHS) in 2002 - 2003, around $4 \%$ of women of childbearing age use traditional family planning (KB) tools/methods, which increased to $5 \%$ in the 2007 IDHS. In the 2012 IDHS, there was a decline in the trend of using traditional contraceptives, to $4 \%$, but there was an increase again, reaching $6 \%$, in the 2017 IDHS [10]. In the Special Region of Yogyakarta Province, the proportion of traditional contraceptive users, based on the 2017 IDHS, reached $18 \%$ of the total users of contraception of all methods, indicating an increasing trend when compared to the 2012 IDHS results, which were around $10 \%$. Based on the 2017 IDHS, it is known that the use of traditional contraceptives is higher in urban areas than in rural areas. The impact of the use of traditional contraceptives contributes significantly to the occurrence of unwanted pregnancies, especially in developing countries [10,16]. The Central Bureau of Statistics (BPS) also reported that the use of traditional contraceptives contributed to nearly $30 \%$ to cases of unwanted pregnancy [2].

This study aims to describe the correlation between predisposing, enabling, and reinforcing factors in family planning access among the urban poor in the City of Yogyakarta. This theory is then elaborated on using the precede-proceed model [18]. According to the previous explanation of Green theory, the behavior of individuals or groups in accessing health services is determined by 3 factors: Predisposing, enabling and reinforcing factors [19].

\section{Materials and methods}

\section{Data sources}

This study uses probability sampling to draw conclusions (inference). The sampling technique used was the cluster sampling technique. The number of samples for each region is described in Figure 1, below:

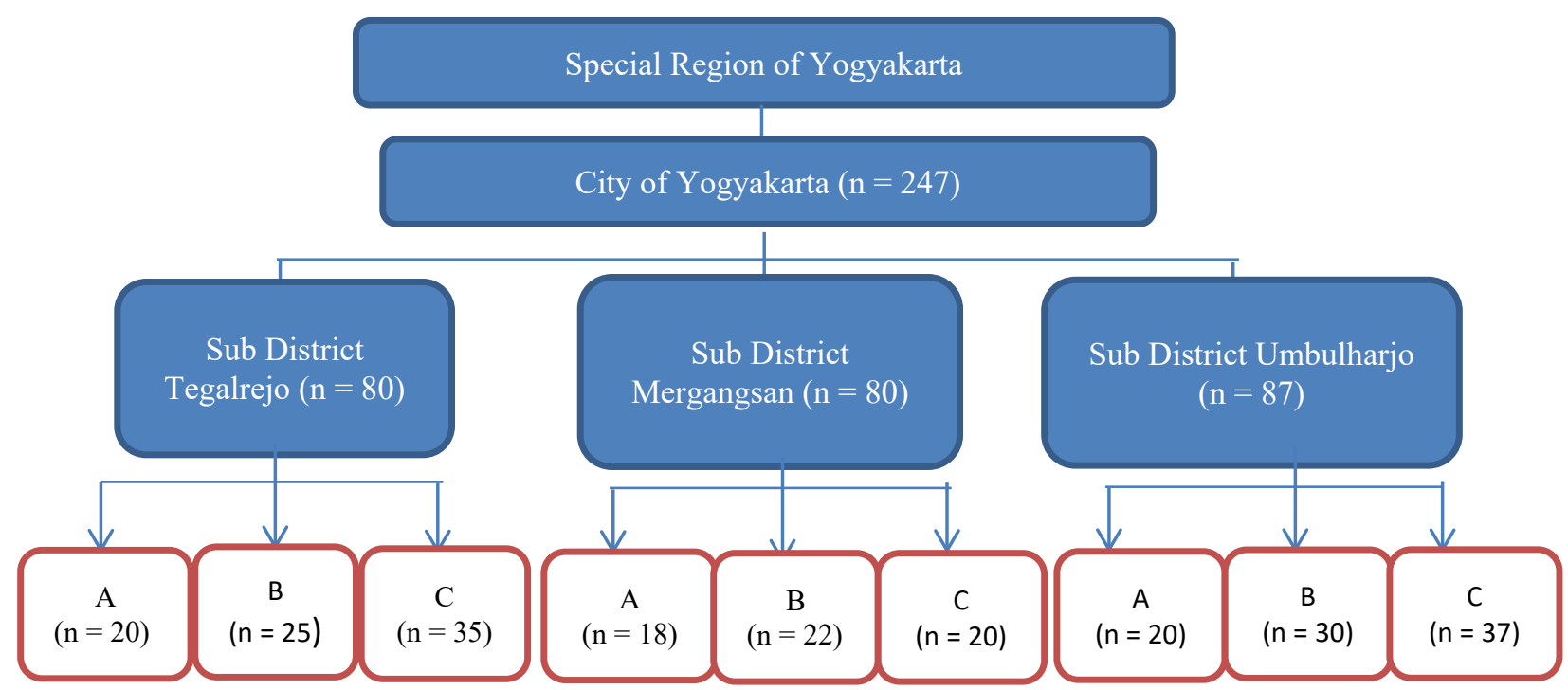

Figure 1 Sample frame. 
The sample of this study was of married women of childbearing age, aged 15 - 49 years. The research sample met the inclusion criteria, namely, living in the Tegalrejo, Mergangsang, or Umbulharjo areas for at least 6 months, being poor, and being willing to be the respondents of this study. This research was voluntary, so that if a respondent refused to be interviewed, the researcher would replace them with another respondent. None of the respondents who were invited refused to be interviewed. All of the respondents were given a souvenir after the interview session, to express our thanks for their time and information in this study. This research instrument used a questionnaire, which was an improvement from the 2017 Indonesian Demographic and Health Survey questionnaire, which consisted of 6 parts, namely the socio-demography of respondents, knowledge of family planning, contraceptive use behavior, and access to family planning services, family support, friends and health workers. In order to maintain the confidentiality of informant data, informed consent was conducted prior to conducting interviews.

\section{Data analysis}

Access to family planning services meant health facilities accessed by poor women in an effort to obtain contraceptive services. In this study, access to family planning services was categorized as midwives, health centers, clinics, government hospitals, private hospitals, mobile family planning services and pharmacies.

Independent variables of this study were age, education level, employment status, family income, knowledge of family planning, number of children, family support, support from friends, support from health workers, family planning visits, and national health insurance or Jaminan Kesehatan Nasional (JKN) membership. All variables involved in the analysis of this study were dichotomous; therefore, the chi square test was used to determine whether there was a meaningful correlation in choosing family planning services among poor child-bearing aged women in the city of Yogyakarta.

Frequency distribution was done to identify all responses. In addition, this was followed by bivariate statistical analysis (cross tabulation) to identify the relationship between the dependent and independent variables to obtain candidate variables $(p<0.25)$ which would be used as predictors in multivariate analysis (multiple logistic regression). All statistical analyses of quantitative results were carried out with the help of STATA version 13.0.

\section{Ethical statement}

The Ahmad Dahlan University ethics committee approved the ethical clearance of this study. The respondents' identities have all been deleted from the dataset. Respondents provided written approval for their involvement in the study. Researchers obtained data collection permits and dissemination of research results on ethics certificate number 011801013.

\section{Abbreviation}

Bappenas: The Ministry of National Development Planning (National Development Planning Agency); BKKBN: National Population and Family Planning Agency; JKN: Jaminan Kesehatan Nasional (National Health Insurance); MoW: Metode Operasi Wanita (Tubectomy); PHC: Public Health Centre (pusat kesehatan masyarakat or puskesmas).

\section{Results and discussion}

\section{Socio-demographics of respondents}

Table 1 explains that the largest age group of respondents was aged 40 - 44 years, comprising 64 people $(25.01 \%)$. The majority of respondents had completed high school education, at a number of 114 people $(46.15 \%)$. More than half of respondents are unemployed, at 144 people $(58.29 \%)$. The majority of respondents had a family income of $<1,700,000$ IDR (USD 110.6), at 173 people (70.04\%). The majority of respondents had good contraception knowledge, namely 192 people $(77.73 \%)$. The number of children owned, by $189(76.51 \%)$ respondents, was $\leq 2$ children. The majority of respondents, totaling 209 people $(84.61 \%)$, received family support to use contraception. The majority of respondents, totaling 206 people $(83.40 \%)$, received the support of friends to use contraception. 232 people (93.92\%) stated that they received support from health workers to use contraception. The majority of respondents, 230 people $(93.12 \%)$, said they had never been visited by family planning officers. A total of 232 respondents $(93.93 \%)$ had health insurance. 
Table 1 Socio-demographics of the respondents $(n=247)$.

\begin{tabular}{|c|c|c|c|}
\hline Variables & Responses & Freq. & Percent \\
\hline \multirow[t]{6}{*}{ Age } & $<25$ years & 7 & 2.83 \\
\hline & $25-29$ years & 22 & 8.90 \\
\hline & $30-34$ years & 57 & 23.07 \\
\hline & 35 - 39 years & 57 & 23.07 \\
\hline & 40 - 44 years & 64 & 25.91 \\
\hline & $45-49$ years & 40 & 16.19 \\
\hline \multirow[t]{5}{*}{ Level of education } & No Education & 7 & 2.83 \\
\hline & Primary & 39 & 15.79 \\
\hline & Junior high school & 68 & 27.53 \\
\hline & Senior high school & 114 & 46.15 \\
\hline & Undergraduate & 19 & 7.69 \\
\hline \multirow[t]{2}{*}{ Job status } & Unemployment & 144 & 58.29 \\
\hline & Employment & 103 & 41.70 \\
\hline \multirow[t]{2}{*}{ Family income } & $<1,700,000 \mathrm{IDR}$ & 173 & 70.04 \\
\hline & $\geq 1,700,000 \mathrm{IDR}$ & 74 & 29.95 \\
\hline \multirow[t]{3}{*}{ Knowledge of FP } & Less & 5 & 2.02 \\
\hline & Average & 50 & 20.24 \\
\hline & Good & 192 & 77.73 \\
\hline \multirow[t]{2}{*}{ Number of children } & $\leq 2$ & 189 & 76.51 \\
\hline & $>2$ & 58 & 23.48 \\
\hline \multirow[t]{3}{*}{ Family support } & Unsupported & 34 & 13.76 \\
\hline & Supported & 209 & 84.61 \\
\hline & Neutral & 4 & 1.62 \\
\hline \multirow[t]{3}{*}{ Peer support } & Unsupported & 19 & 7.69 \\
\hline & Support & 206 & 83.40 \\
\hline & Neutral & 22 & 8.91 \\
\hline \multirow[t]{3}{*}{ Health providers support } & Unsupported & 10 & 4.04 \\
\hline & Supported & 232 & 93.92 \\
\hline & Neutral & 5 & 2.02 \\
\hline \multirow[t]{2}{*}{ Visited by FP officer } & No & 230 & 93.12 \\
\hline & Yes & 17 & 6.88 \\
\hline \multirow[t]{2}{*}{ Health insurance Membership } & Not membership & 15 & 6.07 \\
\hline & Membership & 232 & 93.93 \\
\hline
\end{tabular}

\section{Bivariate analysis}

Table 2 is a bivariate statistic between predisposing factors (age, level of education, job status and number of children), enabling factors (family income and knowledge of FP), and reinforcing factors (family support, peer support, health provider support, FP officer having visited, and health insurance membership). Table 2 explains that women aged $25-29$ years had a tendency to access family planning services in public health centers (PHC) or puskesmas, with a proportion of 0.688 ( $p$-value $0.648,95 \% \mathrm{CI}$ : $0.434-0.864)$. Women with the level of education of having graduated from college chose to access family planning services at PHCs with a proportion of 0.809 ( $p$-value $0.015,95 \%$ CI $0.564-0.933$ ). Women who did not work chose to access family planning services in clinics with a proportion of 0.704 ( $p$-value $0.162,95 \%$ CI $0.616-0.780$ ). Women with an income of $\geq 1,700,000$ IDR chose to access family planning services in clinics with a proportion of 0.702 . Women who had knowledge about family planning chose to access family planning services in PHCs with a proportion of 0.700 .

Table 2 shows that women who had a number of children $\leq 2$ chose to access family planning services at PHCs with a proportion of 0.684 . Women with families who were not supported chose access to family planning services in PHCs with a proportion of 0.754 ( $p$-value $0.148,95 \%$ CI $0.565-0.887$ ). 
Women with peers who supported them preferred to choose the place of family planning services in PHCs with a proportion of 0.713 ( $p$-value $0.028,95 \%$ CI 0.639 - 0.777 ). Women who received support from health workers chose access to family planning services in health clinics with a proportion of 0.689 and with statistical significance ( $p$-value $0.325,95 \%$ CI $0.617-0.752)$. Women who were not visited by family planning officers chose to access family planning services in midwives with a proportion of 0.154 ( $p$-value $0.508,95 \%$ CI 0.109 - 0.214).

Table 2 shows that women visited by family planning officers chose to access family planning services in clinics with a proportion of 0.778 ( $p$-value $0.508,95 \%$ CI $0.419-0.927$ ). Women who had national health insurance or Jaminan Kesehatan Nasional (JKN) cards chose to access family planning services from midwives with a proportion of 150 and with statistical significance ( $p$-value $0.909,95 \% \mathrm{CI}$ $0.106-0.209$ ). Women who did not have a JKN card chose to access family planning services in clinics with a proportion of 0.805 and were statistically significant ( $p$-value $0.909,95 \%$ CI 0.473 - 0.950 ). Table 2 explains that the factors related to the selection of places to gain access to family planning services for the urban poor in Yogyakarta City was peer support with a $p$-value $=0.028$.

The results showed that the places to access family planning services in the city of Yogyakarta for the urban poor group consisted of midwives, public health centers, health clinics, government hospitals, private hospitals and family planning on mobile services. Based on age, preference for places to obtain family planning services for the urban poor in the city of Yogyakarta was as follows: Age $<25$ years preferred to access family planning services in PHCs and Health Clinics, 25 - 29 years of age preferred visiting private hospitals to access family planning services, 30 - 34 years preferred accessing mobile services, 40 - 44 years preferred accessing midwives, and 45 - 49 years preferred accessing government hospitals and dispensaries, even though age categories were not significantly associated with accessing family planning services.

Women who had graduated tertiary education level chose to access family planning services at PHCs with a proportion of 0.809 ( $p$-value $0.015,95 \%$ CI $0.564-0.933$ ). However, the results of this study indicate that the level of education did not correlate with the choice of family planning services among the urban poor in Yogyakarta. This finding is different from the results of previous studies, which stated that the level of education was related to the experience of a person or group in choosing health $[20,21]$. Women with an income of $\geq 1,700,000$ IDR chose to access family planning services in clinics with a proportion of 0.702 . The results of this study are in line with research that explained that a person's financial ability determines the choosing of the type of health service. The lack of a person's financial ability then has a tendency to not access health services or to choose unpaid health services [22,23].

Women with friends who supported them preferred to access family planning services in PHCs with a proportion of 0.713 ( $p$-value $0.028,95 \%$ CI $0.639-0.777$ ). Peer support had a strong correlation in influencing a person's attitudes and behaviors to make decisions in choosing contraceptive services. Friends are individuals and groups that influence very strongly someone's decision-making behavior to act. In general, friends have the same experience, so this strengthens individuals to make decisions $[18,21]$.

Based on the results of the research that has been done, it is shown that friends are a factor influencing someone to choose a contraceptive method in the urban poor group. Thus, it is very important to educate couples of childbearing age by initiating the formation of family planning care groups as peer educators, as well as strengthening family planning cadres in each region as family planning facilitators. This research becomes richer if it is deepened by conducting a qualitative study exploring the reasons for using traditional contraceptives from social and religious perspectives. 
Table 2 Correlation between predisposing, enabling, and reinforcing factors and access of family planning services among urban poor in Yogyakarta.

\begin{tabular}{|c|c|c|c|c|c|c|c|c|c|c|c|c|c|c|c|}
\hline \multirow{3}{*}{ Variable } & \multicolumn{15}{|c|}{ Access of family planning services } \\
\hline & \multicolumn{2}{|c|}{ Midwives } & \multicolumn{2}{|c|}{ PHC } & \multicolumn{2}{|c|}{ Health clinic } & \multicolumn{2}{|c|}{ Gov. hosp } & \multicolumn{2}{|c|}{ Private hosp } & \multicolumn{2}{|c|}{ Mobile service } & \multicolumn{2}{|c|}{ Drug store } & \multirow{2}{*}{$\begin{array}{l}\text { Total } \\
\text { Prop }\end{array}$} \\
\hline & Prop & $95 \% \mathrm{CI}$ & Prop & $95 \% \mathrm{CI}$ & Prop & $95 \% \mathrm{CI}$ & Prop & $95 \% \mathrm{CI}$ & Prop & $95 \% \mathrm{CI}$ & Prop & $95 \% \mathrm{CI}$ & Prop & $95 \% \mathrm{CI}$ & \\
\hline \multicolumn{16}{|l|}{ Age } \\
\hline $\begin{array}{c}<25 \text { years } \\
(\mathrm{n}=7)\end{array}$ & 0.000 & & 0.794 & $\begin{array}{c}{[0.308-} \\
0.971]\end{array}$ & 0.206 & $\begin{array}{c}{[0.029-} \\
0.692]\end{array}$ & 0.000 & & 0.000 & & 0.000 & & 0.000 & & 1.000 \\
\hline $\begin{array}{c}25-29 \text { years } \\
(\mathrm{n}=22)\end{array}$ & 0.156 & $\begin{array}{l}{[0.041-} \\
0.443]\end{array}$ & 0.688 & $\begin{array}{c}{[0.434-} \\
0.864]\end{array}$ & 0.015 & $\begin{array}{c}{[0.002-} \\
0.107]\end{array}$ & 0.047 & $\begin{array}{c}{[0.006-} \\
0.275]\end{array}$ & 0.070 & $\begin{array}{c}{[0.010-} \\
0.366]\end{array}$ & 0.000 & & 0.024 & $\begin{array}{c}{[0.003-} \\
0.156]\end{array}$ & 1.000 \\
\hline $\begin{array}{c}30-34 \text { years } \\
(\mathrm{n}=57)\end{array}$ & 0.175 & $\begin{array}{c}{[0.091-} \\
0.309]\end{array}$ & 0.630 & $\begin{array}{c}{[0.484-} \\
0.756]\end{array}$ & 0.077 & $\begin{array}{c}{[0.027-} \\
0.205]\end{array}$ & 0.014 & $\begin{array}{c}{[0.002-} \\
0.097]\end{array}$ & 0.000 & & 0.029 & $\begin{array}{c}{[0.004-} \\
0.180]\end{array}$ & 0.074 & $\begin{array}{c}{[0.026-} \\
0.195]\end{array}$ & 1.000 \\
\hline $\begin{array}{c}35-39 \text { years } \\
(\mathrm{n}=57)\end{array}$ & 0.119 & $\begin{array}{c}{[0.057-} \\
0.234]\end{array}$ & 0.791 & $\begin{array}{c}{[0.656-} \\
0.883]\end{array}$ & 0.024 & $\begin{array}{c}{[0.003-} \\
0.154]\end{array}$ & 0.021 & $\begin{array}{c}{[0.003-} \\
0.139]\end{array}$ & 0.028 & $\begin{array}{c}{[0.004-} \\
0.175]\end{array}$ & 0.011 & $\begin{array}{c}{[0.001-} \\
0.073]\end{array}$ & 0.005 & $\begin{array}{c}{[0.001-} \\
0.038]\end{array}$ & 1.000 \\
\hline $\begin{array}{c}40-44 \text { years } \\
(n=64)\end{array}$ & 0.189 & $\begin{array}{c}{[0.103-} \\
0.322]\end{array}$ & 0.629 & $\begin{array}{c}{[0.487-} \\
0.752]\end{array}$ & 0.068 & $\begin{array}{c}{[0.023-} \\
0.183]\end{array}$ & 0.031 & $\begin{array}{c}{[0.007-} \\
0.123]\end{array}$ & 0.050 & $\begin{array}{c}{[0.012-} \\
0.181]\end{array}$ & 0.000 & & 0.033 & $\begin{array}{c}{[0.007-} \\
0.136]\end{array}$ & 1.000 \\
\hline $\begin{array}{c}45-49 \text { years } \\
(\mathrm{n}=40)\end{array}$ & 0.101 & $\begin{array}{c}{[0.034-} \\
0.263]\end{array}$ & 0.654 & $\begin{array}{c}{[0.468-} \\
0.802]\end{array}$ & 0.039 & $\begin{array}{c}{[0.005-} \\
0.234]\end{array}$ & 0.094 & $\begin{array}{c}{[0.027-} \\
0.278]\end{array}$ & 0.000 & & 0.014 & $\begin{array}{c}{[0.002-} \\
0.096]\end{array}$ & 0.098 & $\begin{array}{c}{[0.030-} \\
0.278]\end{array}$ & 1.000 \\
\hline Total $(\mathrm{n}=247)$ & 0.148 & $\begin{array}{c}{[0.105-} \\
0.204]\end{array}$ & 0.681 & $\begin{array}{c}{[0.612-} \\
0.743]\end{array}$ & 0.056 & $\begin{array}{c}{[0.031-} \\
0.102]\end{array}$ & 0.034 & $\begin{array}{c}{[0.016-} \\
0.069]\end{array}$ & 0.025 & $\begin{array}{c}{[0.009-} \\
0.066]\end{array}$ & 0.012 & $\begin{array}{c}{[0.003-} \\
0.043]\end{array}$ & 0.044 & $\begin{array}{c}{[0.023-} \\
0.084]\end{array}$ & 1.000 \\
\hline \multicolumn{16}{|c|}{$\begin{array}{c}\text { Pearson: Uncorrected chi2 }(30)=30.424 \\
\text { Design-based } \mathrm{F}(27.10,6667.47)=0.877 \\
\quad p \text {-value }=0.648\end{array}$} \\
\hline \multicolumn{16}{|l|}{$\begin{array}{c}\text { Level of } \\
\text { education }\end{array}$} \\
\hline $\begin{array}{c}\text { Uneducated SD (n } \\
=7 \text { ) }\end{array}$ & 0.188 & $\begin{array}{c}{[0.026-} \\
0.665]\end{array}$ & 0.321 & $\begin{array}{c}{[0.086-} \\
0.703]\end{array}$ & 0.000 & & 0.308 & $\begin{array}{c}{[0.075-} \\
0.708]\end{array}$ & 0.000 & & 0.000 & & 0.183 & $\begin{array}{c}{[0.025-} \\
0.659]\end{array}$ & 1.000 \\
\hline Primary $(\mathrm{n}=39)$ & 0.155 & $\begin{array}{c}{[0.063-} \\
0.333]\end{array}$ & 0.660 & $\begin{array}{c}{[0.474-} \\
0.807]\end{array}$ & 0.089 & $\begin{array}{c}{[0.025-} \\
0.273]\end{array}$ & 0.059 & $\begin{array}{c}{[0.014-} \\
0.213]\end{array}$ & 0.000 & & 0.000 & & 0.038 & $\begin{array}{c}{[0.005-} \\
0.226]\end{array}$ & 1.000 \\
\hline $\begin{array}{l}\text { Junior high school } \\
\qquad(\mathrm{n}=68)\end{array}$ & 0.184 & $\begin{array}{c}{[0.100-} \\
0.312]\end{array}$ & 0.685 & $\begin{array}{c}{[0.550-} \\
0.795]\end{array}$ & 0.033 & $\begin{array}{c}{[0.008-} \\
0.127]\end{array}$ & 0.017 & $\begin{array}{c}{[0.004-} \\
0.066]\end{array}$ & 0.000 & & 0.018 & $\begin{array}{c}{[0.004-} \\
0.071]\end{array}$ & 0.063 & $\begin{array}{c}{[0.021-} \\
0.175]\end{array}$ & 1.000 \\
\hline $\begin{array}{l}\text { Senior high school } \\
\qquad(\mathrm{n}=114)\end{array}$ & 0.131 & $\begin{array}{c}{[0.077-} \\
0.212]\end{array}$ & 0.693 & $\begin{array}{c}{[0.590-} \\
0.780]\end{array}$ & 0.063 & $\begin{array}{c}{[0.026-} \\
0.142]\end{array}$ & 0.018 & $\begin{array}{c}{[0.004-} \\
0.072]\end{array}$ & 0.053 & $\begin{array}{c}{[0.020-} \\
0.133]\end{array}$ & 0.015 & $\begin{array}{c}{[0.002-} \\
0.101]\end{array}$ & 0.027 & $\begin{array}{c}{[0.009-} \\
0.079]\end{array}$ & 1.000 \\
\hline $\begin{array}{c}\text { Undergraduate } \\
\quad(\mathrm{n}=19)\end{array}$ & 0.103 & $\begin{array}{c}{[0.023-} \\
0.357]\end{array}$ & 0.809 & $\begin{array}{c}{[0.564-} \\
0.933]\end{array}$ & 0.055 & $\begin{array}{c}{[0.008-} \\
0.311]\end{array}$ & 0.000 & & 0.000 & & 0.000 & & 0.033 & $\begin{array}{c}{[0.004-} \\
0.208]\end{array}$ & 1.000 \\
\hline Total $(n=247)$ & 0.148 & $\begin{array}{c}{[0.105-} \\
0.204]\end{array}$ & 0.681 & $\begin{array}{c}{[0.612-} \\
0.743]\end{array}$ & 0.056 & $\begin{array}{c}{[0.031-} \\
0.102]\end{array}$ & 0.034 & $\begin{array}{c}{[0.016-} \\
0.069]\end{array}$ & 0.025 & $\begin{array}{c}{[0.009-} \\
0.066]\end{array}$ & 0.012 & $\begin{array}{c}{[0.003-} \\
0.043]\end{array}$ & 0.044 & $\begin{array}{c}{[0.023-} \\
0.084]\end{array}$ & 1.000 \\
\hline \multicolumn{16}{|c|}{$\begin{array}{c}\text { Pearson: Uncorrected chi2 }(24)=42.465 \\
\text { Design-based } \mathrm{F}(20.54,5052.09)=1.562 \\
p \text {-value }=0.051\end{array}$} \\
\hline Job status & & & & & & & & & & & & & & & \\
\hline $\begin{array}{l}\text { Unemployed } \\
\quad(\mathrm{n}=144)\end{array}$ & 0.156 & $\begin{array}{c}{[0.102-} \\
0.231]\end{array}$ & 0.704 & $\begin{array}{c}{[0.616-} \\
0.780]\end{array}$ & 0.054 & $\begin{array}{c}{[0.025-} \\
0.116]\end{array}$ & 0.007 & $\begin{array}{c}{[0.001-} \\
0.040]\end{array}$ & 0.029 & $\begin{array}{c}{[0.009-} \\
0.086]\end{array}$ & 0.004 & $\begin{array}{c}{[0.001-} \\
0.028]\end{array}$ & 0.046 & $\begin{array}{c}{[0.019-} \\
0.106]\end{array}$ & 1.000 \\
\hline $\begin{array}{l}\text { Employed } \\
(\mathrm{n}=103)\end{array}$ & 0.135 & $\begin{array}{c}{[0.075-} \\
0.231]\end{array}$ & 0.644 & $\begin{array}{c}{[0.530-} \\
0.744]\end{array}$ & 0.059 & $\begin{array}{c}{[0.022-} \\
0.150]\end{array}$ & 0.077 & $\begin{array}{c}{[0.035-} \\
0.161]\end{array}$ & 0.019 & $\begin{array}{c}{[0.003-} \\
0.125]\end{array}$ & 0.024 & $\begin{array}{c}{[0.005-} \\
0.111]\end{array}$ & 0.042 & $\begin{array}{c}{[0.016-} \\
0.106]\end{array}$ & 1.000 \\
\hline Total $(\mathrm{n}=247)$ & 0.148 & $\begin{array}{c}{[0.105-} \\
0.204]\end{array}$ & 0.681 & $\begin{array}{c}{[0.612-} \\
0.743]\end{array}$ & 0.056 & $\begin{array}{c}{[0.031-} \\
0.102]\end{array}$ & 0.034 & $\begin{array}{c}{[0.016-} \\
0.069]\end{array}$ & 0.025 & $\begin{array}{c}{[0.009-} \\
0.066]\end{array}$ & 0.012 & $\begin{array}{c}{[0.003-} \\
0.043]\end{array}$ & 0.044 & $\begin{array}{c}{[0.023-} \\
0.084]\end{array}$ & 1.000 \\
\hline \multicolumn{16}{|c|}{$\begin{array}{c}\text { Pearson: Uncorrected chi2 }(6)=11.147 \\
\text { Design-based } \mathrm{F}(5.63,1385.51)=1.552 \\
p \text {-value }=0.162\end{array}$} \\
\hline $\begin{array}{c}\text { Family income } \\
<1.700 .000 \text { IDR } \\
(\mathrm{n}=173)\end{array}$ & 0.162 & $\begin{array}{c}{[0.110-} \\
0.232]\end{array}$ & 0.673 & $\begin{array}{c}{[0.590-} \\
0.746]\end{array}$ & 0.033 & $\begin{array}{c}{[0.013-} \\
0.078]\end{array}$ & 0.038 & $\begin{array}{c}{[0.017-} \\
0.086]\end{array}$ & 0.035 & $\begin{array}{c}{[0.013-} \\
0.091]\end{array}$ & 0.006 & $\begin{array}{c}{[0.002-} \\
0.026]\end{array}$ & 0.052 & $\begin{array}{c}{[0.025-} \\
0.107]\end{array}$ & 1.000 \\
\hline $\begin{array}{c}\geq 1.700 .000 \text { IDR } \\
(\mathrm{n}=74)\end{array}$ & 0.112 & $\begin{array}{c}{[0.052-} \\
0.223]\end{array}$ & 0.702 & $\begin{array}{c}{[0.568-} \\
0.808]\end{array}$ & 0.114 & $\begin{array}{c}{[0.050-} \\
0.240]\end{array}$ & 0.023 & $\begin{array}{c}{[0.005-} \\
0.101]\end{array}$ & 0.000 & & 0.025 & $\begin{array}{c}{[0.003-} \\
0.158]\end{array}$ & 0.025 & $\begin{array}{c}{[0.007-} \\
0.082]\end{array}$ & 1.000 \\
\hline Total $(\mathrm{n}=247)$ & 0.148 & $\begin{array}{c}{[0.105-} \\
0.204]\end{array}$ & 0.681 & $\begin{array}{c}{[0.612-} \\
0.743]\end{array}$ & 0.056 & $\begin{array}{c}{[0.031-} \\
0.102]\end{array}$ & 0.034 & $\begin{array}{c}{[0.016-} \\
0.069]\end{array}$ & 0.025 & $\begin{array}{c}{[0.009-} \\
0.066]\end{array}$ & 0.012 & $\begin{array}{c}{[0.003-} \\
0.043]\end{array}$ & 0.044 & $\begin{array}{c}{[0.023-} \\
0.084]\end{array}$ & 1.000 \\
\hline \multicolumn{16}{|c|}{$\begin{array}{c}\text { Pearson: Uncorrected chi2 }(6)=12.104 \\
\text { Design-based } \mathrm{F}(5.78,1423.02)=1.737 \\
\quad p \text {-value }=0.112\end{array}$} \\
\hline \multicolumn{16}{|l|}{ Knowledge of FP } \\
\hline Less $(n=5)$ & 0.516 & $\begin{array}{c}{[0.146-} \\
0.869]\end{array}$ & 0.484 & $\begin{array}{c}{[0.131-} \\
0.854]\end{array}$ & 0.000 & & 0.000 & & 0.000 & & 0.000 & & 0.000 & & 1.000 \\
\hline Average $(\mathrm{n}=50)$ & 0.228 & $\begin{array}{c}{[0.122-} \\
0.387]\end{array}$ & 0.633 & $\begin{array}{c}{[0.473-} \\
0.769]\end{array}$ & 0.060 & $\begin{array}{c}{[0.017-} \\
0.191]\end{array}$ & 0.051 & $\begin{array}{c}{[0.013-} \\
0.184]\end{array}$ & 0.027 & $\begin{array}{c}{[0.004-} \\
0.171]\end{array}$ & 0.000 & & 0.000 & & 1.000 \\
\hline Good $(n=192)$ & 0.115 & $\begin{array}{c}{[0.075-} \\
0.173]\end{array}$ & 0.700 & $\begin{array}{c}{[0.621-} \\
0.768]\end{array}$ & 0.057 & $\begin{array}{c}{[0.028-} \\
0.112]\end{array}$ & 0.030 & $\begin{array}{c}{[0.013-} \\
0.071]\end{array}$ & 0.025 & $\begin{array}{c}{[0.008-} \\
0.076]\end{array}$ & 0.015 & $\begin{array}{c}{[0.004-} \\
0.056]\end{array}$ & 0.057 & $\begin{array}{c}{[0.030-} \\
0.107]\end{array}$ & 1.000 \\
\hline Total $(\mathrm{n}=247)$ & 0.148 & $\begin{array}{c}{[0.105-} \\
0.204]\end{array}$ & 0.681 & $\begin{array}{c}{[0.612-} \\
0.743]\end{array}$ & 0.056 & $\begin{array}{c}{[0.031-} \\
0.102]\end{array}$ & 0.034 & $\begin{array}{c}{[0.016-} \\
0.069]\end{array}$ & 0.025 & $\begin{array}{c}{[0.009-} \\
0.066]\end{array}$ & 0.012 & $\begin{array}{c}{[0.003-} \\
0.043]\end{array}$ & 0.044 & $\begin{array}{c}{[0.023-} \\
0.084]\end{array}$ & 1.000 \\
\hline \multirow{3}{*}{\multicolumn{16}{|c|}{$\begin{array}{c}\text { Pearson: Uncorrected chi2 }(12)=14.915 \\
\text { Design-based } \mathrm{F}(10.83,2663.08)=1.045 \\
\quad p \text {-value }=0.403\end{array}$}} \\
\hline & & & & & & & & & & & & & & & \\
\hline & & & & & & & & & & & & & & & \\
\hline $\begin{array}{c}\text { Number of } \\
\text { children }\end{array}$ & & & & & & & & & & & & & & & \\
\hline $\begin{array}{c}\leq 2 \text { Children } \\
(\mathrm{n}=189)\end{array}$ & 0.147 & $\begin{array}{c}{[0.100-} \\
0.213]\end{array}$ & 0.684 & $\begin{array}{c}{[0.605-} \\
0.755]\end{array}$ & 0.062 & $\begin{array}{c}{[0.031-} \\
0.118]\end{array}$ & 0.024 & $\begin{array}{c}{[0.009-} \\
0.062]\end{array}$ & 0.026 & $\begin{array}{c}{[0.008-} \\
0.079]\end{array}$ & 0.006 & $\begin{array}{c}{[0.001-} \\
0.024]\end{array}$ & 0.051 & $\begin{array}{c}{[0.025-} \\
0.100]\end{array}$ & 1.000 \\
\hline $\begin{array}{c}>2 \text { Children } \\
\quad(\mathrm{n}=58)\end{array}$ & 0.148 & $\begin{array}{c}{[0.071-} \\
0.283]\end{array}$ & 0.671 & $\begin{array}{c}{[0.524-} \\
0.791]\end{array}$ & 0.041 & $\begin{array}{c}{[0.010-} \\
0.151]\end{array}$ & 0.065 & $\begin{array}{c}{[0.022-} \\
0.179]\end{array}$ & 0.022 & $\begin{array}{c}{[0.003-} \\
0.142]\end{array}$ & 0.029 & $\begin{array}{c}{[0.004-} \\
0.181]\end{array}$ & 0.024 & $\begin{array}{c}{[0.003-} \\
0.152]\end{array}$ & 1.000 \\
\hline Total $(n=247)$ & 0.148 & {$[0.105-$} & 0.681 & {$[0.612-$} & 0.056 & {$[0.031-$} & 0.034 & {$[0.016-$} & 0.025 & {$[0.009$ - } & 0.012 & {$[0.003-$} & 0.044 & {$[0.023-$} & 1.000 \\
\hline
\end{tabular}




\begin{tabular}{|c|c|c|c|c|c|c|c|c|c|c|c|c|c|c|c|}
\hline \multirow{3}{*}{ Variable } & \multicolumn{15}{|c|}{ Access of family planning services } \\
\hline & \multicolumn{2}{|c|}{ Midwives } & \multicolumn{2}{|c|}{ PHC } & \multicolumn{2}{|c|}{ Health clinic } & \multicolumn{2}{|c|}{ Gov. hosp } & \multicolumn{2}{|c|}{ Private hosp } & \multicolumn{2}{|c|}{ Mobile service } & \multicolumn{2}{|c|}{ Drug store } & \multirow{2}{*}{$\begin{array}{l}\text { Total } \\
\text { Prop }\end{array}$} \\
\hline & Prop & $95 \% \mathrm{CI}$ & Prop & $95 \% \mathrm{CI}$ & Prop & $95 \% \mathrm{CI}$ & Prop & $95 \% \mathrm{CI}$ & Prop & $95 \% \mathrm{CI}$ & Prop & $95 \% \mathrm{CI}$ & Prop & $95 \% \mathrm{CI}$ & \\
\hline & & $0.204]$ & & $0.743]$ & & $0.102]$ & & $0.069]$ & & $0.066]$ & & $0.043]$ & & $0.084]$ & \\
\hline \multicolumn{16}{|c|}{ Pearson: Uncorrected chi2 $(6)=5.640$} \\
\hline \multicolumn{16}{|c|}{ Design-based $\mathrm{F}(5.94,1460.09)=0.737$} \\
\hline \multicolumn{16}{|c|}{$\mathrm{P}$-value $=0,619$} \\
\hline \multicolumn{16}{|l|}{ Family support } \\
\hline $\begin{array}{l}\text { Unsupported } \\
(\mathrm{n}=34)\end{array}$ & 0.064 & $\begin{array}{c}{[0.016-} \\
0.223]\end{array}$ & 0.754 & $\begin{array}{c}{[0.565-} \\
0.878]\end{array}$ & 0.086 & $\begin{array}{c}{[0.024-} \\
0.264]\end{array}$ & 0.056 & $\begin{array}{c}{[0.013-} \\
0.211]\end{array}$ & 0.041 & $\begin{array}{c}{[0.006-} \\
0.241]\end{array}$ & 0.000 & & 0.000 & & 1.000 \\
\hline $\begin{array}{l}\text { Supported } \\
(\mathrm{n}=209)\end{array}$ & 0.163 & $\begin{array}{c}{[0.115-} \\
0.227]\end{array}$ & 0.672 & $\begin{array}{c}{[0.596-} \\
0.740]\end{array}$ & 0.052 & $\begin{array}{c}{[0.026-} \\
0.103]\end{array}$ & 0.031 & $\begin{array}{c}{[0.013-} \\
0.070]\end{array}$ & 0.023 & $\begin{array}{c}{[0.007-} \\
0.069]\end{array}$ & 0.014 & $\begin{array}{c}{[0.004-} \\
0.051]\end{array}$ & 0.045 & $\begin{array}{c}{[0.022-} \\
0.089]\end{array}$ & 1.000 \\
\hline Neutral $(n=4)$ & 0.000 & & 0.482 & $\begin{array}{c}{[0.082-} \\
0.907]\end{array}$ & 0.000 & & 0.000 & & 0.000 & & 0.000 & & 0.518 & $\begin{array}{c}{[0.093-} \\
0.918]\end{array}$ & 1.000 \\
\hline Total $(n=247)$ & 0.148 & $\begin{array}{c}{[0.105-} \\
0.204] \\
\end{array}$ & 0.681 & $\begin{array}{c}{[0.612-} \\
0.743] \\
\end{array}$ & 0.056 & $\begin{array}{c}{[0.031-} \\
0.102] \\
\end{array}$ & 0.034 & $\begin{array}{c}{[0.016-} \\
0.069] \\
\end{array}$ & 0.025 & $\begin{array}{c}{[0.009-} \\
0.066] \\
\end{array}$ & 0.012 & $\begin{array}{c}{[0.003-} \\
0.043] \\
\end{array}$ & 0.044 & $\begin{array}{c}{[0.023-} \\
0.084] \\
\end{array}$ & 1.000 \\
\hline \multicolumn{16}{|c|}{ Pearson: Uncorrected chi2 $(12)=21.040$} \\
\hline \multicolumn{16}{|c|}{ Design-based $F(11.52,2832.96)=1.430$} \\
\hline \multicolumn{16}{|c|}{$p$-value $=0.148$} \\
\hline \multicolumn{16}{|l|}{ Peer support } \\
\hline $\begin{array}{l}\text { Unsupported } \\
\quad(\mathrm{n}=19)\end{array}$ & 0.222 & $\begin{array}{c}{[0.082-} \\
0.477]\end{array}$ & 0.545 & $\begin{array}{c}{[0.309-} \\
0.763]\end{array}$ & 0.163 & $\begin{array}{c}{[0.051-} \\
0.415]\end{array}$ & 0.000 & & 0.070 & $\begin{array}{c}{[0.010-} \\
0.366]\end{array}$ & 0.000 & & 0.000 & & 1.000 \\
\hline $\begin{array}{l}\text { Supported } \\
(\mathrm{n}=206)\end{array}$ & 0.147 & $\begin{array}{c}{[0.101-} \\
0.210]\end{array}$ & 0.713 & $\begin{array}{c}{[0.639-} \\
0.778]\end{array}$ & 0.031 & $\begin{array}{c}{[0.012-} \\
0.077]\end{array}$ & 0.034 & $\begin{array}{c}{[0.016-} \\
0.074]\end{array}$ & 0.023 & $\begin{array}{c}{[0.007-} \\
0.070]\end{array}$ & 0.014 & $\begin{array}{c}{[0.004-} \\
0.052]\end{array}$ & 0.037 & $\begin{array}{c}{[0.018-} \\
0.075]\end{array}$ & 1.000 \\
\hline Neutral $(n=22)$ & 0.080 & $\begin{array}{c}{[0.018-} \\
0.293]\end{array}$ & 0.486 & $\begin{array}{c}{[0.262-} \\
0.715]\end{array}$ & 0.212 & $\begin{array}{c}{[0.071-} \\
0.487]\end{array}$ & 0.061 & $\begin{array}{c}{[0.008-} \\
0.333]\end{array}$ & 0.000 & & 0.000 & & 0.161 & $\begin{array}{c}{[0.041-} \\
0.462]\end{array}$ & 1.000 \\
\hline Total $(n=247)$ & 0.148 & $\begin{array}{c}{[0.105-} \\
0.204]\end{array}$ & 0.681 & $\begin{array}{c}{[0.612-} \\
0.743]\end{array}$ & 0.056 & $\begin{array}{c}{[0.031-} \\
0.102] \\
\end{array}$ & 0.034 & $\begin{array}{c}{[0.016-} \\
0.069]\end{array}$ & 0.025 & $\begin{array}{c}{[0.009-} \\
0.066]\end{array}$ & 0.012 & $\begin{array}{c}{[0.003-} \\
0.043]\end{array}$ & 0.044 & $\begin{array}{c}{[0.023-} \\
0.084]\end{array}$ & 1.000 \\
\hline & & & & & & arson: Unce & rected $\mathrm{cl}$ & $i 2(12)=29$ & 632 & & & & & & \\
\hline & & & & & & sign-based & $(11.72,2$ & $884.06)=1$ & & & & & & & \\
\hline & & & & & & & alue $=0$ & 028 & & & & & & & \\
\hline $\begin{array}{l}\text { Health provider } \\
\text { Support }\end{array}$ & & & & & & & & & & & & & & & \\
\hline $\begin{array}{l}\text { Unsupported } \\
(\mathrm{n}=10)\end{array}$ & 0.090 & $\begin{array}{c}{[0.012-} \\
0.447]\end{array}$ & 0.638 & $\begin{array}{c}{[0.297-} \\
0.880]\end{array}$ & 0.142 & $\begin{array}{c}{[0.020-} \\
0.575]\end{array}$ & 0.000 & & 0.130 & $\begin{array}{c}{[0.018-} \\
0.550]\end{array}$ & 0.000 & & 0.000 & & 1.000 \\
\hline $\begin{array}{l}\text { Supported } \\
(\mathrm{n}=232)\end{array}$ & 0.147 & $\begin{array}{c}{[0.103-} \\
0.206]\end{array}$ & 0.689 & $\begin{array}{c}{[0.617-} \\
0.752]\end{array}$ & 0.054 & $\begin{array}{c}{[0.028-} \\
0.100]\end{array}$ & 0.036 & $\begin{array}{c}{[0.017-} \\
0.074]\end{array}$ & 0.021 & $\begin{array}{c}{[0.007-} \\
0.063]\end{array}$ & 0.013 & $\begin{array}{c}{[0.003-} \\
0.046]\end{array}$ & 0.041 & $\begin{array}{c}{[0.020-} \\
0.081]\end{array}$ & 1.000 \\
\hline $\begin{array}{l}\text { Neutral } \\
(\mathrm{n}=5)\end{array}$ & 0.278 & $\begin{array}{c}{[0.038-} \\
0.790]\end{array}$ & 0.427 & $\begin{array}{c}{[0.096-} \\
0.840]\end{array}$ & 0.000 & & 0.000 & & 0.000 & & 0.000 & & 0.295 & $\begin{array}{c}{[0.041-} \\
0.803]\end{array}$ & 1.000 \\
\hline Total $(n=247)$ & 0.148 & $\begin{array}{c}{[0.105-} \\
0.204] \\
\end{array}$ & 0.681 & $\begin{array}{c}{[0.612-} \\
0.743] \\
\end{array}$ & 0.056 & $\begin{array}{c}{[0.031-} \\
0.102] \\
\end{array}$ & 0.034 & $\begin{array}{c}{[0.016-} \\
0.069]\end{array}$ & 0.025 & $\begin{array}{c}{[0.009-} \\
0.066]\end{array}$ & 0.012 & $\begin{array}{c}{[0.003-} \\
0.043]\end{array}$ & 0.044 & $\begin{array}{c}{[0.023-} \\
0.084] \\
\end{array}$ & 1.000 \\
\hline & & & & & & arson: Unce & rected cl & $i 2(12)=16$ & & & & & & & \\
\hline & & & & & & sign-based & $(10.78,2$ & $652.27)=1$ & 141 & & & & & & \\
\hline & & & & & & & alue $=0$ & 325 & & & & & & & \\
\hline FP officer visited & & & & & & & & & & & & & & & \\
\hline No $(n=230)$ & 0.154 & $\begin{array}{c}{[0.109-} \\
0.214]\end{array}$ & 0.673 & $\begin{array}{c}{[0.601-} \\
0.738]\end{array}$ & 0.061 & $\begin{array}{c}{[0.033-} \\
0.110]\end{array}$ & 0.030 & $\begin{array}{c}{[0.014-} \\
0.063]\end{array}$ & 0.021 & $\begin{array}{c}{[0.007-} \\
0.064]\end{array}$ & 0.013 & $\begin{array}{c}{[0.003-} \\
0.047]\end{array}$ & 0.048 & $\begin{array}{c}{[0.025-} \\
0.090]\end{array}$ & 1.000 \\
\hline Yes $(n=17)$ & 0.062 & $\begin{array}{c}{[0.009-} \\
0.340]\end{array}$ & 0.778 & $\begin{array}{c}{[0.491-} \\
0.927]\end{array}$ & 0.000 & & 0.087 & $\begin{array}{c}{[0.012-} \\
0.424]\end{array}$ & 0.073 & $\begin{array}{c}{[0.010-} \\
0.377]\end{array}$ & 0.000 & & 0.000 & & 1.000 \\
\hline Total $(n=247)$ & 0.148 & $\begin{array}{c}{[0.105-} \\
0.204]\end{array}$ & 0.681 & $\begin{array}{c}{[0.612-} \\
0.743]\end{array}$ & 0.056 & $\begin{array}{c}{[0.031-} \\
0.102]\end{array}$ & 0.034 & $\begin{array}{c}{[0.016-} \\
0.069]\end{array}$ & 0.025 & $\begin{array}{c}{[0.009-} \\
0.066]\end{array}$ & 0.012 & $\begin{array}{c}{[0.003-} \\
0.043]\end{array}$ & 0.044 & $\begin{array}{c}{[0.023-} \\
0.084]\end{array}$ & 1.000 \\
\hline & & & & & & earson: Un & rrected & $\operatorname{hi} 2(6)=6.9$ & & & & & & & \\
\hline & & & & & & sign-based & $(5.96,1$ & $65.20)=0$ & & & & & & & \\
\hline & & & & & & & alue $=0$ & & & & & & & & \\
\hline $\begin{array}{l}\text { Health insurance } \\
\text { Membership }\end{array}$ & & & & & & & & & & & & & & & \\
\hline $\begin{array}{l}\text { Not membership } \\
\quad(\mathrm{n}=15)\end{array}$ & 0.103 & $\begin{array}{c}{[0.015-} \\
0.474]\end{array}$ & 0.805 & $\begin{array}{c}{[0.473-} \\
0.950]\end{array}$ & 0.092 & $\begin{array}{c}{[0.013-} \\
0.442]\end{array}$ & 0.000 & & 0.000 & & 0.000 & & 0.000 & & 1.000 \\
\hline $\begin{array}{l}\text { Membership } \\
(\mathrm{n}=232)\end{array}$ & 0.150 & $\begin{array}{c}{[0.106-} \\
0.209]\end{array}$ & 0.673 & $\begin{array}{c}{[0.601-} \\
0.737]\end{array}$ & 0.054 & $\begin{array}{c}{[0.028-} \\
0.101]\end{array}$ & 0.036 & $\begin{array}{c}{[0.017-} \\
0.074]\end{array}$ & 0.027 & $\begin{array}{c}{[0.010-} \\
0.070]\end{array}$ & 0.013 & $\begin{array}{c}{[0.003-} \\
0.046]\end{array}$ & 0.047 & $\begin{array}{c}{[0.024-} \\
0.089]\end{array}$ & 1.000 \\
\hline Total $(n=247)$ & 0.148 & $\begin{array}{c}{[0.105-} \\
0.204] \\
\end{array}$ & 0.681 & $\begin{array}{c}{[0.612-} \\
0.743] \\
\end{array}$ & 0.056 & $\begin{array}{c}{[0.031-} \\
0.102] \\
\end{array}$ & 0.034 & $\begin{array}{c}{[0.016-} \\
0.069] \\
\end{array}$ & 0.025 & $\begin{array}{c}{[0.009-} \\
0.066] \\
\end{array}$ & 0.012 & $\begin{array}{c}{[0.003-} \\
0.043] \\
\end{array}$ & 0.044 & $\begin{array}{c}{[0.023-} \\
0.084] \\
\end{array}$ & 1.000 \\
\hline & & & & & & earson: Un & rrected & $\operatorname{hi} 2(6)=2.8$ & & & & & & & \\
\hline & & & & & & sign-based & $(5.93,1$ & $58.25)=0$ & & & & & & & \\
\hline & & & & & & & alue $=0$ & & & & & & & & \\
\hline & & & & & & Source: & rimary & ata, 2018 & & & & & & & \\
\hline
\end{tabular}




\section{Conclusions}

Based on the results of the data analysis, it can be concluded that the factor affecting family planning program access among poor couples in the city of Yogyakarta is peer support.

\section{Acknowledgements}

The author thanks the National Population and Family Planning Agency (BKKBN) for funding the research and supporting the publication of this article (Grant Certificate Number: 2596/PL.201/PK/H4/VII/2018). The funding agency had no role in designing the study, conducting the analysis, interpreting the data or writing the manuscript.

\section{References}

[1] NDP Agency. Performance report 2018. vol. 53. NDP Agency, 2018.

[2] BPS-Statistics Indonesia. Statistical yearbook of Indonesia 2018. BPS-Statistics Indonesia, Jakarta, 2018.

[3] F Götmark and $M$ Andersson. Human fertility in relation to education, economy, religion, contraception, and family planning programs. BMC Publ. Health 2020; 20, 265.

[4] A Kabagenyi, P Kakande and V Owayezu. Demand for family planning among poor women in Uganda: Analysis of the Uganda demographic and health surveys. DHS Working Paper, Maryland, 2020, p. 1-19.

[5] JK Wulifan, J Mazalale, A Jahn, H Hien, PC Ilboudo, N Meda, PJ Robyn, S Hamadou, O Haidara and MD Allegri. Factors associated with contraceptive use among women of reproductive age in Rural districts of Burkina Faso. J. Health Care Poor Underserved 2017; 28, 228-47.

[6] S Thongmixay, T Schoneveld, V Vongxay, JEW Broerse, V Sychareun and DR Essink. Quality of family planning services for women of reproductive age in Lao PDR. Glob. Health Action 2020; 13, 1788261.

[7] A Kabagenyi, P Kakande and V Owayezu. Demand for family planning among poor women in Uganda: Analysis of the Uganda demographic and health surveys. Res. Square 2020, DOI: 10.21203/rs.3.rs-39847/v1.

[8] SA Wilopo and AW Pinandari. Position and chance of Indonesia family planning to achieve RPJMN 2015 - 2019 and FP 2020 targets. J. Kesehatan Masyarakat 2013; 9, 37-43.

[9] Y Amran, NM Nasir, D Dachlia, F Yelda, B Utomo, I Ariawan and R Damayanti. Perceptions of contraception and patterns of switching contraceptive methods among family-planning acceptors in West Nusa Tenggara, Indonesia. J. Prev. Med. Publ. Health 2019; 52, 258-64.

[10] National Population and Family Planning Board (BKKBN), Statistics Indonesia (BPS), Ministry of Health (Kemenkes) and ICF. Indonesia demographic and health survey 2017. BKKBN, BPS, Kemenkes and ICF, Jakarta, 2018.

[11] M Kadarisman. Family planning program in the national population and family planning board. Adv. Soc. Sci. Educ. Humanit. Res. 2019; 343, 266-9.

[12] B Abdi, J Okal, G Serour and M Temmerman. Children are a blessing from God - a qualitative study exploring the socio - cultural factors influencing contraceptive use in two Muslim communities in Kenya. Reprod. Health 2020; 17, 44.

[13] K Elmusharaf, E Byrne and D O’Donovan. Social and traditional practices and their implications for family planning: A participatory ethnographic study in Renk, South Sudan. Reprod. Health 2017; 14, 10.

[14] R Stephenson and M Hennink. Barriers to family planning service use among the urban poor in Pakistan. Asia Pac. Popul. J. 2004; 19, 5-26.

[15] AC Ezeh, I Kodzi and J Emina. Reaching the urban poor with family planning services. Stud. Fam. Plann. 2010; 41, 109-16.

[16] F Ewerling, CG Victora, A Raj, CVN Coll, F Hellwig and AJD Barros. Demand for family planning satisfied with modern methods among sexually active women in low- and middle-income countries: Who is lagging behind? Reprod. Health 2018; 15, 42.

[17] NL Price and K Hawkins. A conceptual framework for the social analysis of reproductive health. $J$. Health Popul. Nutr. 2007; 25, 24-36.

[18] LW Green and JM Ottoson. A framework for planning and evaluation: PRECEDE-PROCEED evolution and application of the model. 10Es Ans Journees Sante Publique, 2006, p. 1-4.

[19] RM Andersen. Revisiting the behavioral model and access to medical care: Does it matter? $J$. 
Health Soc. Behav. 1995; 36, 1-10.

[20] A Gafar, DE Suza, F Efendi, EMM Has, AP Pramono and IA Susanti. Determinants of contraceptive use among married women in Indonesia. F1000Research 2020; 9, 193.

[21] N Rosmala, A Rahman and S Krisnasari. Factors associated with the use of non-hormonal contraceptive methods in the Pantoloan public health center Palu City. J. Curr. Med. Res. Opin. $2019 ; 2,115-20$.

[22] M Ali, SK Azmat, HB Hamza, MM Rahman and W Hameed. Are family planning vouchers effective in increasing use, improving equity and reaching the underserved? An evaluation of a voucher program in Pakistan. BMC Health Serv. Res. 2019; 19, 200.

[23] A Kumar, AK Jain, Kumudha Aruldas, Arupendra Mozumdar, A Shukla and R Acharya. Is economic inequality in family planning in India associated with the private sector? J. Biosoc. Sci. 2019. 52, 248-59. 